\title{
Specific Antibody Deficiency in Pediatric Patients with Chronic Rhinosinusitis
}

\author{
Robert G Hill, BA ${ }^{1}$ and James A Sipp, $M D^{2^{*}}$ \\ ${ }^{1}$ Mercer University School of Medicine, USA \\ ${ }^{2}$ ENT of Georgia, USA \\ *Corresponding author: James A Sipp, ENT of Georgia, USA
}

\begin{abstract}
Objectives: This study was performed to review our experience with pediatric patients adaptive immunity to Streptococcus pneumoniae and Haemophilus influenza in the clinical presentation of chronic rhinosinusitis. Currently, there is no specific routine role in healthcare to check the immune status in patients after receiving the vaccinations.
\end{abstract}

Methods: Patients were identified from the EMR using the ICD-10 code for chronic sinusitis and were considered eligible if they had titers drawn to evaluate their immunity. Patients being reviewed were between 2 and 18 years of age. None had a history of global immunological disease, cystic fibrosis, craniofacial abnormalities, or nasal polyps. Their vaccination records were verified using the state record system, GRITS, or by patient intake history. Titers were then compared to the geometric mean established by previous academic research and the minimum level recommended for protective immunity as outlined by the WHO.

Results: We found that in a sample of 36 patients that met the criteria for this study, $76 \%$ had S. pneumoniae titers that fell below the reported geometric mean of $1.3 \mathrm{mcg} / \mathrm{mL}$. Similarly, $45 \%$ of the patients had $H$. influenza titers that fell below the reported geometric mean of $1.0 \mathrm{mcg} / \mathrm{mL}$. Both of these values were found to be statistically significant. There was no significant relationship present between the immunogenicity rates of the vaccines and the minimum level required for protective immunity as outlined by the World Health Organization.

Conclusion: Specific antibody deficiency to $S$. pneumoniae and $H$. influenza pathogens in pediatric patients with chronic rhinosinusitis had a high prevalence.

\section{Introduction}

Nearly all pediatric otolaryngologists struggle to treat a handful of patients with chronic rhinosinusitis (CRS). In a recent analysis of national surveys, CRS was estimated to account for 3.7-7.5 million visits per year in patients 0 to 20 years of age and the burden exceeded that of acute rhinosinusitis and allergic rhinitis [1]. First line medical treatment for these patients has traditionally involved long duration antibiotics coupled with short term corticosteroids and treatment of allergic symptoms versus allergy evaluation [2]. Following conservative treatment, if refractory disease is still present patients will typically be evaluated for an adenoidectomy or other sinus surgery depending on the extent of the disease [3]. While these treatments are effective, there continue to be patients who struggle controlling their symptoms even after going through surgery [4]. Two of the most common bacterial agents that cause sinusitis are $S$. pneumoniae and $H$. influenza [5], however, since the introduction of Pneumococcal and Hib vaccines, the frequencies of these invasive pathologies have decreased drastically [6-8]. Immunogenicity rates for PCV 7 and 13 have been reported at $89 \%$ for children who receive the recommended doses with the geometric mean being observed at $1.3 \mathrm{mcg} / \mathrm{mL}$ [7]. Hib vaccination has similarly been found to have a high rate of immunogenicity, $90 \%$ in a 5 year study [6].

Recent publications have begun analyzing the burden of specific antibody deficiencies (SAD) and their impact on healthcare. SAD is grossly defined as having an antibody concentration lower than expected at a certain age and is most often discussed in its relation to S. pneumoniae. Analysis of IgG serotypes is performed quantitatively but remains somewhat arbitrary values where $0.35 \mathrm{mcg} / \mathrm{mL}$ is considered protective against invasive disease and $1.3 \mathrm{mcg} / \mathrm{mL}$ is recognized as pro-

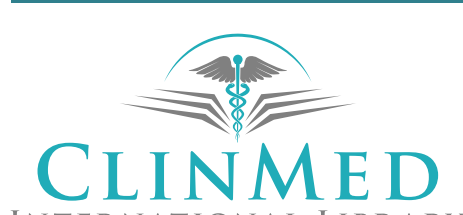

INTERNATIONAL LIBRARY
Citation: Hill RG, Sipp JA (2021) Specific Antibody Deficiency in Pediatric Patients with Chronic Rhinosinusitis. Int J Blood Res Disord 8:061. doi.org/10.23937/2469-5696/1410061

Accepted: February 17, 2021: Published: February 19, 2021

Copyright: (C) 2021 Hill RG, et al. This is an open-access article distributed under the terms of the Creative Commons Attribution License, which permits unrestricted use, distribution, and reproduction in any medium, provided the original author and source are credited. 
tective against mucosal diseases [9]. Interpretation of these results can become even more complicated when analyzing as $50-70 \%$ of the serotypes should be above the geometric mean to be considered immunocompetent; however, not all patients with SAD are symptomatic [10]. A retrospective study of adult patients with SAD observed that $77 \%$ suffered from sinusitis as well as other recurrent infections [11]. The exact prevalence of SAD remains unclear as not all are symptomatic as stated above, but studies within the pediatric population estimate it to be $15-23 \%[12,13]$. Evidence in one study found that $11.6 \%$ of patients undergoing sinus surgery also had SAD, however, this was a study of an adult population [14]. To our knowledge, similar studies have not been performed for $\mathrm{H}$. influenzae.

While a large number of patients who do not mount an adequate immune response to these pathogens will develop an invasive disease or be found to be globally immunodeficient, statistically a certain population will show a "sub-optimal" response to vaccination or exposure. Currently there is no specific routine role in healthcare to check the immune response in patients after receiving vaccinations. We propose that if we review a sample population of pediatric patients with chronic rhinosinusitis, then their reported titers to $S$. pneumoniae and $H$. influenza will be statistically lower than previously reported responses.

\section{Methods}

This is a retrospective analysis of pediatric patient's adaptive immunity to $S$. pneumoniae and $H$. influenza in the clinical presentation of chronic rhinosinusitis over a 24 month period (December 2015 to December 2017) in Atlanta, Georgia by a single pediatric otolaryngologist. Once Institutional Review Board approval was obtained, patients were identified by searching the EMR based off of the ICD-10 code for chronic sinusitis, J32.9. Originally, 61 patients were identified in the time frame that was diagnosed with chronic sinusitis. 25 patients were then excluded from the analysis because they did not fall within the age range of 2 to 18 years of age at the time of the blood draw, they did not have their blood drawn at all, or did not have a viable vaccination record. None had a history of global immunological disease, cystic fibrosis, craniofacial abnormalities, or nasal polyps. Of the 36 patients remaining, their vaccination records were verified using the state record system, GRITS, or by patient intake history. Of note, two of the patients had a history significant for Down Syndrome and were included in this report. Once their blood work was obtained from either LabCorp or Quest, the common 14 strand pneumococcal titer $(1,3,4,8,9,12,14,19,23$, $26,51,56,57$, and 68 ) that overlapped both labs were compared against the minimum value that the WHO recommends for immunity against invasive pneumococcal disease as well as the geometric mean, with values set at 0.35 and $1.3 \mathrm{mcg} / \mathrm{mL}$ respectively [15]. A pa- tient was considered to be sub-optimal if half of their 14 pneumococcal strands were below the geometric mean $(<1.3 \mathrm{mcg} / \mathrm{mL})$. If half were below $0.35 \mathrm{mcg} / \mathrm{mL}$ they were considered to not have any protective immunity as outlined by the WHO.

$H$. Influenza titers were reviewed in a similar manner, however, due to a lab coding error, of the 36 patients only 29 in the sample had viable lab values. The same exclusion criteria was used for $H$. influenzae as was for $S$. pneumoniae. When analyzing the type B antibody concentrations for $H$. influenzae, below $0.15 \mathrm{mcg} /$ $\mathrm{mL}$ was considered non-protective and between 0.15 $\mathrm{mcg} / \mathrm{mL}$ and $1.0 \mathrm{mcg} / \mathrm{mL}$ was considered sub-optimal. Above $1.0 \mathrm{mcg} / \mathrm{mL}$ was considered to have protective immunity as outlined from the WHO and previous studies $[8,16]$.

\section{Results}

\section{Streptococcus pneumoniae}

In this retrospective analysis 26 out of 36 patients (73\%) had suboptimal immunity levels, defined as having at least 7 of the 14 tested strains below the geometric mean of $1.3 \mathrm{mcg} / \mathrm{mL}$. In this group, 6 out of 36 patients $(17 \%)$ were considered to have no immunity, defined as having at least half of their titers were below the WHO's recommended minimum level of 0.35 $\mathrm{mcg} / \mathrm{mL}$. There was no observed statistically significant difference between the reported immunogenicity rates of PCV and our sample population. When analyzing the total number of patients below the geometric mean (sub-optimal + non-immune $=26$ of the 36 patients) against previously reported sample sizes, there was a statistically significant difference using chi-square analysis $(p=0.05)[5]$

\section{Haemophilus influenza}

Twenty-nine of the patients had viable $H$. influenza titers available. Of this sample, 16 out of 29 (55\%) were considered optimal whereas the remaining 13 (45\%) were below $1.0 \mathrm{mcg} / \mathrm{mL}$ and therefore suboptimal. Of those remaining patients, 7 (24\%) were considered to not have protective immunity $(<0.15 \mathrm{mcg} / \mathrm{mL})$. When compared to predictive larger sample sizes as referenced in previous studies, the number of patients below $0.15 \mathrm{mcg} / \mathrm{mL}$ was statistically significant using chisquare analysis $(p=0.05)[6]$.

\section{Discussion}

While we were able to find a statistically significant difference between our sample population when compared to reference values provided by the World Health Organization and other reputable academic sources, a better control group would be to have age-matched controls from the same population. In particular, we found it difficult to run basic statistical analysis for the $H$. influenza sample as available literature provided proportions and not sample sizes. Predictive sample sizes 
were estimated to analyze this sample and aid in the interpretation of results and discussion. An attempt was made to analyze the specific serotypes of $S$. pneumoniae against the specific strains that were covered in respective vaccines. This data was deemed non-valuable as many patients received a combination of vaccines and the specific overlap of reported lab serotypes did not provide significant overlap.

Although the sample population was small, SAD had a high prevalence in both $S$. pneumoniae and $H$. influen$z a$ groups when compared to the immunogenicity rates of their respective vaccines. This suggests that there could be an immunological component to pediatric patients with chronic rhinosinusitis. As researchers, we admit this data was difficult to compare because of the lack of prior research on this topic specifically. We do however feel that it warrants the assessment of a patient's immunological status at some point in the management of chronic rhinosinusitis.

This study did not measure outcomes in a systematic way and therefore does not provide evidence in regard to recommendations on maintenance vaccinations, booster vaccinations, or other treatment options. Future goals of research should include: (1) Analysis with age appropriate control groups, (2) Outcome studies to assess response rates to different treatment plans, and (3) Systematic reviews to assess for iatrogenic risk.

\section{Conclusion}

Selective antibody deficiency to $S$. pneumoniae and $H$. influenza pathogens in pediatric patients with chronic rhinosinusitis had a high prevalence. Outcome studies are warranted to assess the role of checking immunity to $S$. pneumoniae and $H$. influenza in the management of patients with chronic rhinosinusitis as well as administering Pneumovax (PPS-23), Prevnar 13 (PCV 13), and/ or Hib vaccine booster.

\section{Conflicts of Interest}

We do not have any conflicts of interest to disclose.

\section{References}

1. Gilani S, Shin JJ (2017) The burden and visit prevalence of pediatric chronic rhinosinusitis. Otolaryngol Head Neck Surg 157: 1048-1052.

2. Beswick DM, Messner AH, Hwang PH (2017) Pediatric chronic rhinosinusitis management in rhinologists and pediatric otolaryngologists. Ann Otol Rhinol Laryngol 126: 634-639.
3. Gudis DA, Soler ZM (2017) Update on pediatric sinus surgery: Indications and outcomes. Curr Opin Otolaryngol Head Neck Surg 25: 486-492.

4. Beswick DM, Ramadan H, Baroody FM, Hwang PH (2016) Practice patterns in pediatric chronic rhinosinusitis: $A$ survey of the American Rhinologic Society. Am J Rhinol Allergy 30: 418-423.

5. Brook I (2007) Acute and chronic bacterial sinusitis. Infect Dis Clin North Am 21: 427-448.

6. Zarei AE, Almehdar HA, Redwan EM (2016) Hib vaccines: Past, present, and future perspectives. J Immunol Res.

7. Ruiz-Aragon J, Marquez Pelaez S, Molina-Linde JM, Grande-Tejada AM (2013) Safety and immunogenicity of 13-valent pneumococcal conjugate vaccine in infants: A meta-analysis. Vaccine 31: 5349-5358.

8. Robbins JB, Parke JC, Schneerson R, Whisnant JK (1973) Quantitative measurement of "natural" and immunization-induced Haemophilus influenzae type b capsular polysaccharide antibodies. Pediatr Res 7: 103-110.

9. Sorensen RU, Edgar D (2019) Specific antibody deficiencies in clinical practice. J Allergy Clin Immunol Pract 7: 801808.

10. Stevens WW, Peters AT (2015) Immunodeficiency in chronic sinusitis: Recognition and treatment. Am J Rhinol Allergy 29: 115-118.

11. Cheng YK, Decker PA, O'Byrne MM, Weiler CR (2006) Clinical and laboratory characteristics of 75 patients with specific polysaccharide antibody deficiency syndrome. Ann Allergy Asthma Immunol Off Publ Am Coll Allergy Asthma Immunol 97: 306-311.

12. Javier FC, Moore CM, Sorensen RU (2000) Distribution of primary immunodeficiency diseases diagnosed in a pediatric tertiary hospital. Ann Allergy Asthma Immunol Off Publ Am Coll Allergy Asthma Immunol 84: 25-30.

13. Boyle RJ, Le C, Balloch A, Tang ML-K (2006) The clinical syndrome of specific antibody deficiency in children. Clin Exp Immunol 146: 486-492.

14. Carr TF, Koterba AP, Chandra R, Grammer LC, Conley DB, et al. Characterization of specific antibody deficiency in adults with medically refractory chronic rhinosinusitis. Am J Rhinol Allergy 25: 241-244.

15. Bryant K, Frenck RW (2016) Response to letter to the editor regarding: Immunogenicity and safety of a 13-valent pneumococcal conjugate vaccine in adults $18-49$ years of age, naive to 23-valent pneumococcal polysaccharide vaccine. Vaccine.

16. Anderson P (1984) The protective level of serum antibodies to the capsular polysaccharide of Haemophilus influenzae type b. J Infect Dis 149: 1034-1035. 